\title{
Avoiding "curiosity killed the cat": personalized surveillance in post-resection colorectal cancer patients
}

Hyunkyung Park and Joohan Lim

Department of Internal Medicine, Inha University School of

Medicine, Incheon, Korea

Received: June 12, 2018

Accepted: June 18, 2018

\section{Correspondence to}

Joohan Lim, M.D.

Department of Internal Medicine, Inha University School of Medicine, 27 Inhang-ro, Junggu, Incheon 22332, Korea

Tel: $+82-32-890-3498$

Fax: +82-32-890-2582

E-mail:limjh@inha.ac.kr

\section{See Article on Page 783-789}

Colorectal cancer (CRC) is the third most common cancer globally and imposes a significant burden on healthcare. The disease is the second leading cause of cancer-related death in the United States, accounting for more than 50,000 deaths annually [1]. In South Korea, the incidence of CRC has increased in recent years, becoming the fourth-most-common cause of cancer-related death, with the second highest incidence rate among malignant neoplasms in 2015 [2]. For an average risk individual, the lifetime risk of eventually developing CRC is upwards of $5 \%$.

Surgical resection is the primary and the only curative treatment in patients with resectable CRC. Despite surgery and the adjuvant chemotherapy and/or radiation treatment, about $40 \%$ of patients with stage II or III CRC patients will have recurrence of the disease. In CRC, $80 \%$ of recurrences occurred in the first 3 years [3], with $95 \%$ of recurrences occurring in the first 5 years after surgical resection of a primary tumor [4].

For patients with resectable synchronous or metachronous oligometastatic $\mathrm{CRC}$, there are potentially curative treatments. These treatments may also extend to patients with early-detected metachronous oligometastatic CRC. Moreover, improvement in overall survival may be achieved through early detection of recurrent disease, particularly in isolated local or distant recurrence, which usually exhibits a higher detection rate. The benefit of surveillance after curative resection of CRC is through early identification of the disease, which may potentially be treated through curative pathways in addition to screening for non-CRC primary cancers. Together, these interventions may help in increasing overall survival for CRC-affected patients.

In the latest issue of Korean Journal of Internal Medicine, Baek et al. [5] reported that the surveillance policies for post-resection CRC implemented by Korean physicians' have a curative aim. They conducted an online prospective questionnaire survey of medical oncologists, surgical oncologists, and gastroenterologist across the country and analyzed the role doctors have in surveillance, examination of surveillance and duration/interval of surveillance according to stage or site. The findings by Baek et al. [5] give relevant information to clinicians in Korea, and could be clinically meaningful despite 
the small number of participants (91 physicians participated, with only 78 completing the survey). According to the authors, physicians in Korea mainly followed up CRC intensively through laboratory tests and computed tomography (CT) scans. For example, $18 \%$ of clinicians who participated the survey monitored patients with stage II/III cancer through CT scans every 3 months, and a further $52 \%$ ordered scans every 6 months during the first year after curative surgery. The Korean physicians who participated in the survey tended to prefer monitoring patients with CRC for at least 5 years after surgical resection, particularly patients with advanced stage, high-risk CRC.

There has yet to be substantial evidence on the optimal surveillance tools, duration, and intervals. Moreover, there are divergent opinions in the guidelines for post-treatment surveillance. For example, The National Comprehensive Cancer Network and European Society of Medical Oncology guidelines recommend abdomen and chest CT surveillance every 6 to 12 months for 3 to 5 years, but The American Society of Clinical Oncology and American Cancer Society recommends CT every 12 months for 5 years. Several randomized controlled trials, meta-analysis, and Cochrane data have been complied to evaluate the efficacy of intensive surveillance strategy for CRC patients after surgery. Some data showed an increased rate of curative-intent surgical treatment with intensive surveillance following the early detection of metachronous metastasis [6-8]. However, studies have not yet demonstrated that intensive surveillance improves overall survival [9,10]. Recently, two separate studies (National Cancer Data Base [NCDB] study [10] from America and the COLOFOL (the pragmatic study to assess the frequency of surveillance tests after curative resection in patients with stage II and II colorectal cancer: a randomized study) trial [11] from Denmark, Sweden, and Uruguay) published in The Journal of the American Medical Association showed that more frequent follow-up testing for patients with CRC did not improve time to recurrence or survival. The authors note, however, that the studies had some limitations in study design. There was also a caution against unnecessary CT scans, which increase costs and radiation exposure to the patients.

The optimal follow-up care regimen for CRC patients has been subjected to an ongoing debate. Data pertaining to stage I or resected stage IV patients are lacking and there are no guidelines for surveillance in the elderly, or when surveillance endoscopies should be stopped.

In summary, more evidence is needed to prove that monitoring CRC patients intensively after curative resection improves overall survival. Overly intensive surveillance such as CT scans every 3 months should be avoided until evidence warrants otherwise. However, considering that the purpose of surveillance of CRC is to identify patients with metachronous disease who may be good candidates for curative resection, intensive surveillance can be justified in high-risk patients and stage IV resected patients.

\section{Conflict of interest}

No potential conflict of interest relevant to this article was reported.

\section{REFERENCES}

1. Siegel RL, Miller KD, Jemal A. Cancer statistics, 2016. CA Cancer J Clin 2016;66:7-30.

2. Jung KW, Won YJ, Kong HJ, Lee ES; Community of Population-Based Regional Cancer Registries. Cancer statistics in Korea: incidence, mortality, survival, and prevalence in 2015. Cancer Res Treat 2018;50:303-316.

3. Sargent D, Sobrero A, Grothey A, et al. Evidence for cure by adjuvant therapy in colon cancer: observations based on individual patient data from 20,898 patients on 18 randomized trials. J Clin Oncol 2009;27:872-877.

4. Seo SI, Lim SB, Yoon YS, et al. Comparison of recurrence patterns between $\leq 5$ years and $>5$ years after curative operations in colorectal cancer patients. J Surg Oncol 2013;108:9-13.

5. Baek SK, Lee SC, Kim JG, et al. Korean physicians' policies for postoperative surveillance of colorectal cancer. Korean J Intern Med 2018;33:783-789.

6. Secco GB, Fardelli R, Gianquinto D, et al. Efficacy and cost of risk-adapted follow-up in patients after colorectal cancer surgery: a prospective, randomized and controlled trial. Eur J Surg Oncol 2002;28:418-423.

7. Rodriguez-Moranta F, Salo J, Arcusa A, et al. Postoperative surveillance in patients with colorectal cancer who have 
undergone curative resection: a prospective, multicenter, randomized, controlled trial. J Clin Oncol 2006;24:386393 .

8. Desch CE, Benson AB 3rd, Somerfield MR, et al. Colorectal cancer surveillance: 2005 update of an American Society of Clinical Oncology practice guideline. J Clin Oncol 2005;23:8512-8519.

9. Primrose JN, Perera R, Gray A, et al. Effect of 3 to 5 years of scheduled CEA and CT follow-up to detect recurrence of colorectal cancer: the FACS randomized clinical trial.
JAMA 2014;311:263-270.

10. Snyder RA, Hu CY, Cuddy A, et al. Association between intensity of posttreatment surveillance testing and detection of recurrence in patients with colorectal cancer. JAMA 2018;319:2104-2115.

11. Wille-Jorgensen P, Syk I, Smedh K, et al. Effect of more vs less frequent follow-up testing on overall and colorectal cancer-specific mortality in patients with stage II or III colorectal cancer: the COLOFOL randomized clinical trial. JAMA 2018;319:2095-2103. 